Advance Publication

\title{
Experimental Animals
}

Received: 2021.3.26

Accepted: 2021.6.17

J-STAGE Advance Published Date: 2021.7.20 
AP39 ameliorates high fat diet-induced liver injury in young rats via. alleviation

Running head: AP39 ALLEVIATES HFD-CAUSED LIVER INJURY

6

Yue $\mathrm{Yu}^{1}$, Shu-Ming Ye ${ }^{2}$, De-Yun Liu ${ }^{1}$, Li-Qi Yang ${ }^{1}{ }^{*}$

$9{ }^{1}$ Department of Pediatrics, The Second Affiliated Hospital of Anhui Medical 10 University, Hefei, Anhui, 230601, China

$11{ }^{2}$ Department of Orthopedics Surgery, The Second Affiliated Hospital of Anhui

*Correspondence to: Li-Qi Yang, Department of Pediatrics, The Second Affiliated Hospital of Anhui Medical University, 678 Furong Road, Hefei, Anhui, 230601, China

17 Telephone: +86-551-65997048 


\section{Abstract}

Non-alcoholic fatty liver disease (NAFLD) is a complication of childhood obesity and an oxidative stress-related multisystem disease. A mitochondria-targeting hydrogen sulfide $\left(\mathrm{H}_{2} \mathrm{~S}\right)$ donor AP39 has antioxidant property, while the mechanism underlying the function of AP39 on pediatric NAFLD remains undefined. Here, 3-week-old SD rats were received a high-fat diet (HFD) feeding and injected with AP39 (0.05 or 0.1 $\mathrm{mg} / \mathrm{kg} /$ day) via the tail vein for up to 7 weeks. AP39 reduced weight gain of HFD rats and improved HFD-caused liver injury, as evidenced by reduced liver index, improved liver pathological damage, decreased NAFLD activity score, as well as low ALT and AST activities. AP39 also reduced serum TC, TG, LDL-C concentrations but increased HDL-C. Moreover, AP39 prevented ROS generation, reduced MDA content and increased GSH level and SOD activity. Furthermore, AP39 increased $\mathrm{H}_{2} \mathrm{~S}$ level, protected mitochondrial DNA (mtDNA), reduced mitochondrial swelling, and restored mitochondrial membrane potential (MMP) alteration. Notably, AP39 diminished HIF-1 $\alpha$ mRNA and protein level, possibly indicating the alleviation in mitochondrial damage. In short, AP39 protects against HFD-induced liver injury in young rats probably through attenuating lipid accumulation, oxidative stress and mitochondrial dysfunction.

Keywords: AP39, HFD, liver injury, oxidative stress, mitochondrial function 


\section{Introduction}

Non-alcoholic fatty liver disease (NAFLD) is a complication of obesity and a main cause of chronic liver disease in children [1]. Its occurrence is associated with insulin resistance, fat accumulation or steatosis, hypertension, dyslipidemia, inflammation, oxidative stress and subsequent hepatotoxicity [2-4]. In recent years, with changes in dietary structure, especially the intake ratio of fats and carbohydrates, the occurrence of NAFLD has increased dramatically, which has been regarded as one of the most prevalent public health problems [5]. There are some therapeutic drugs for NAFLD currently, but considerable side effects and poor long-term safety have been discovered [6]. Therefore, it is very necessary to explore the alternatives available for the effective treatment and prevention of pediatric NAFLD.

Hydrogen sulfide $\left(\mathrm{H}_{2} \mathrm{~S}\right)$, a gaseous signaling molecule, has a variety of biological effects in cellular signaling, including regulating inflammatory response, excessive oxidative stress and apoptosis [7-9]. It has been reported that $\mathrm{H}_{2} \mathrm{~S}$ at a non-cytotoxic level can serve as an electron donor to mitochondria for maintaining the activities of antioxidant enzymes [10]. High-fat diet (HFD) is a leading cause of NAFLD, and $\mathrm{H}_{2} \mathrm{~S}$ alleviates HFD-induced NAFLD in rats [11, 12]. Besides, $\mathrm{H}_{2} \mathrm{~S}$ donor sodium hydrosulfide (NaHS) treatment can also prevent liver injury in rats [13]. These findings indicate that $\mathrm{H}_{2} \mathrm{~S}$ and its relative compounds may have the potential to prevent liver injury.

AP39 (10oxo-10-(4-(3-thioxo-3H-1,2-dithiol-5yl)phenoxy)decyl) is a mitochondria-targeting donor of $\mathrm{H}_{2} \mathrm{~S}$ that can trigger an elevation in $\mathrm{H}_{2} \mathrm{~S}$ production 
in the mitochondria [14]. AP39 has shown antioxidant and other cytoprotective effects under oxidative conditions [15]. Studies have shown that AP39 reduces the release of reactive oxygen species (ROS) and the expression of hypoxia-inducible factor (HIF)-1 $\alpha$ [16]. Also, AP39 has a protective role in damage of multiple organs such as the heart, lung and neuron [17-20]. However, to date, there is no research to clarify whether AP39 can prevent liver injury.

The aim of this study was to investigate the role of AP39 in liver injury in HFD-fed young rats and its underlying mechanisms. Our results demonstrated the protective effect of AP39 on obesity-related liver injury in vivo, providing a theoretical and scientific basis for the use of AP39 in preventing pediatric NAFLD.

\section{Materials and methods}

\section{Animal experiments}

All of our animal experimental protocols were approved by the Institutional Animal Care and Use Committee of Anhui Medical University. Healthy male Sprague-Dawley (SD) rats were purchased from Liaoning Changsheng Biotechnology Co., Ltd. (China). After feeding under controlled laboratory conditions for a week to acclimate to their surroundings, the three-week-old rats were randomly divided into the control group, HFD group, HFD+AP39 low-dose (HFD+L-AP39) and HFD+AP39 high-dose (HFD+H-AP39) groups ( $n=6$ per group).

As shown in Figure 1A, the animals (3 week old) in the control group were fed with a normal fodder (purchased from Beijing Huanyu Zhongke Biotechnology Co., 
Ltd.) for 7 weeks, while the other groups were fed a HFD (69\% of basic feed, $10 \%$ of lard oil, $2 \%$ of cholesterol, $5 \%$ of sugar, $0.5 \%$ of cholate, $10 \%$ of yolk powder, $3 \%$ of yeast powder and $0.5 \%$ of decavitamin; BetterBiotechnology Co., Ltd., Nanjing, China) [21]. To investigate the impact of AP39 on HFD rats, the rats in HFD+L-AP39 and HFD+H-AP39 groups were injected daily by tail vein with AP39 (APExBIO, USA) at the dose of $0.05 \mathrm{mg} / \mathrm{kg}$ or $0.1 \mathrm{mg} / \mathrm{kg}$, respectively [19]. The rats in control and HFD groups were received the same volume of saline. The food intake and body weight of all the rats was recorded per week for up to 7 weeks. At the end of the experiment, all of the tested rats were sacrificed by intraperitoneal injection of overdose $(200 \mathrm{mg} / \mathrm{kg})$ of pentobarbital sodium (Nembutal; Shandong XiYa Chemical Industry Co., Ltd.). Thereafter, the blood was harvested from the inferior vena cava of rats, and the livers were removed, photographed and weighed. Liver weight index was calculated based on the formula liver wet weight / body weight $\times 100 \%$. Tissue samples were either fixed in $4 \%$ paraformaldehyde solution or frozen in liquid nitrogen and stored at $-70^{\circ} \mathrm{C}$ until use.

\section{Histological analysis of liver}

For assessment of lipid staining, the fixed tissues were dehydrated in $20 \%$ and $30 \%$ sucrose solution successively, and embedded with OCT embedding agent, and then sectioned into $10 \mu \mathrm{m}$. The slices were stained with oil red O solution (Sigma, USA) and counterstained with hematoxylin (Solarbio, China). For hematoxylin and eosin (H\&E) staining, the fixed and paraffin-embedded liver samples were cut into $5 \mu \mathrm{m}$ 
slices and stained with hematoxylin and eosin (Sangon, China). All pathological images were observed under an optical microscope at $200 \times$ magnification. After that, based on H\&E staining analysis, the NAFLD activity score, a histological scoring system for NAFLD, was calculated as the unweighted sum of the scores for steatosis (0-3), lobular inflammation (0-3), and ballooning (0-2) [22].

\section{Biochemical parameters}

Serum alanine transaminase (ALT) and aspartate transaminase (AST) activities were determined using the commercial ALT and AST assay kits. Total cholesterol (TC), triglyceride (TG), low-density lipoprotein-cholesterol (LDL-C) and high-density lipoprotein-cholesterol (HDL-C) contents in serum were measured by the TC, TG, LDL-C and HDL-C assay kits, respectively. The levels of oxidative stress parameters malondialdehyde (MDA), glutathione (GSH) and superoxide dismutase (SOD) in liver tissues were examined by the corresponding MDA, GSH and SOD assay kits. The above kits were purchased from Nanjing Jiancheng Bioengineering Institute (China). In addition, hepatic $\mathrm{H}_{2} \mathrm{~S}$ level was measured using a $\mathrm{H}_{2} \mathrm{~S}$ assay kit (Solarbio). All experimental procedures were performed in accordance with the manufacturer's instructions.

\section{DHE staining}

The embedded liver tissues were sectioned into $10 \mu \mathrm{m}$, and incubated with the fluorescent dye dihydroethidium (DHE; Beyotime, China; $1: 100$ dilution) at room temperature for $30 \mathrm{~min}$. The sections were examined using a $400 \times$ fluorescence 
microscope.

\section{Quantitative real-time polymerase chain reaction (RT-qPCR)}

For quantification of $H I F-1 \alpha$, total RNA was extracted using a high-purity total RNA rapid extraction kit (BioTeke, China) and reverse-transcribed into cDNA using the M-MLV reverse transcriptase. Quantitative real-time polymerase chain reaction (RT-qPCR) was then performed on the ExicyclerTM 96 fluorescence quantifier using SYBR Green Master Mix. The relative mRNA expression of $H I F-1 \alpha$ was calculated by the $2^{-\Delta \Delta \mathrm{Ct}}$ method and normalized to $\beta$-actin. For mitochondrial DNA (mtDNA) content determination, DNA extraction was performed using a tissue genomic DNA extraction kit (BioTeke). The copy number of mtDNA was evaluated by RT-qPCR, as described in a previous study [23]. The primers used, synthesized by GenScript (China), were listed in Table 1.

\section{Immunoblot analysis}

Western blot was performed according to Jia et al.'s description [24]. The target protein HIF-1 $\alpha$ was separated, quantified and blotted with anti-HIF-1 $\alpha$ (Affinit, China; $1: 500$ dilution). $\beta$-actin (Proteintech, China; $1: 2000$ dilution) was used for normalization.

\section{Mitochondrial swelling assessment}

Hepatic mitochondria were analyzed using the tissue mitochondrial isolation kit (Beyotime). On the basis of the previous description [25], the degree of liver 
mitochondrial swelling was represented by the relative absorbance value $(\Delta \mathrm{A})$ of mitochondrial suspension at $520 \mathrm{~nm}$ for $20 \mathrm{~min}$. The Larger $\Delta \mathrm{A}$ indicates the more complete mitochondrial structure. A large $\Delta \mathrm{A}$ represents a stronger buffering ability of calcium ions and better structural integrity in mitochondria of liver.

\section{Detection of mitochondrial membrane potential}

Mitochondrial membrane potential (MMP) was detected using the MMP assay kit (Beyotime). The prepared mitochondria were incubated with the fluorescent cationic dye JC-1, followed by analyzing with a fluorescence microplate reader. The red/green fluorescence ratio indicated the activity of hepatic mitochondria.

\section{Statistical analysis}

Quantitative data were reported as mean \pm SD. Statistical analysis was performed with GraphPad Prism 8.0 software. One-way ANOVA followed by Tukey's multiple comparisons test or the Kruskal-Wallis nonparametric analysis of variance followed by Dunn's multiple comparisons test was used for comparative analysis of multiple groups of independent samples. A $p$-value under 0.05 was considered a statistically significant difference.

\section{Results}

\section{Effect of AP39 on body weight}

As illustrated in Figure 1A, the tested young rats with a similar average weight 
were received a HFD feeding and treated with AP39 for 7 weeks. We found that HFD resulted in a significant increase in the body weight of rats compared with normal diet rats. Administration of AP39 reduced HFD-induced weight gain in a dose-dependent manner $(p<0.05$, Figure 1B). In addition, from the 3rd week to the 7 th week of feeding, HFD obviously reduced the food intake of rats, while the food intake of rats with AP39 treatment showed an increasing trend, but it was not significant except for the 5th week (Figure 1C), which was consistent with Jia et al. and Ren et al.' studies $[12,24]$. These findings indicate the potential of AP39 in childhood obesity.

\section{AP39 counteracts HFD-induced liver injury}

Childhood obesity is strongly associated with the occurrence of NAFLD [26]. Here, we focused on the function of AP39 in HFD-caused liver injury in young rats. At the end of the experiment, the livers of the experimental rats were photographed, as shown in Figure 2A. We observed a significant increase in liver size after HFD, with the color changing from dark red to brown yellow, which were improved by AP39 treatment. Moreover, the liver weight index of HFD rats was higher, while AP39 injection partially abolished it ( $p<0.05$, Figure 2B). Histological analysis of the liver using oil red $\mathrm{O}$ (Figure 2C) and H\&E staining (Figure 2D) revealed the changes in hepatic steatosis in rats. We observed that there was increased lipid infiltration area and fatty vacuole area in the liver of HFD rats. However, these liver pathological damages caused by HFD were abrogated and returned to almost normal level after AP39 treatment. NAFLD activity score further confirmed that AP39 
attenuated HFD-induced liver injury ( $p<0.05$, Figure 2E). These findings suggest a beneficial effect of AP39 on HFD-caused liver injury in vivo.

\section{AP39 prevents serum lipid accumulation}

We further verified the lipid-lowing role of AP39, as shown in Figure 3. Serum ALT and AST activities were significantly increased in HFD-induced rats, while AP39 treatment eliminated these changes $(p<0.05$, Figure 3A). Meanwhile, the changes of serum lipid profiles TC, TG, LDL-C and HDL-C were examined. AP39 treatment could induce a reduction in TC, TG, LDL-C levels and an increase in HDL-C in HFD rats $(p<0.05$, Figure 3B-E). Overall, these data suggest that AP39 effectively alleviates lipid accumulation in HFD-fed young rats.

\section{Antioxidant effect of AP39 in NAFLD}

To illuminate the effect of AP39 on oxidative stress, hepatic ROS and oxidative stress-related MDA, GSH, SOD were measured. As visualized by DHE staining, HFD resulted in increased ROS production, which was eliminated by AP39 administration (Figure 4A). In addition, AP39 remarkably decreased MDA content, and increased GSH level and SOD activity in HFD-fed young rats $(p<0.05$, Figure 4B-D), indicating the antioxidant effect of AP39.

\section{AP39 diminishes HFD-caused mitochondrial dysfunction}

As expected, the $\mathrm{H}_{2} \mathrm{~S}$ level in the liver of HFD-fed young rats was lower than that of control rats, while AP39 enhanced it $(p<0.01$, Figure 5A). We then explored 
the role of AP39 in HFD-induced mitochondrial dysfunction. The mtDNA integrity, mitochondrial swelling and MMP alteration were measured to evaluate mitochondrial function. The copy number of mtDNA was increased after AP39 treatment $(p<0.01$, Figure 5B). In addition, AP39 increased the absorbance at $520 \mathrm{~nm}$, suggesting the amelioration of HFD-induced mitochondrial swelling ( $p<0.05$, Figure 5C). The loss of MMP was decreased in AP39-treated HFD rats as compared to HFD-fed rats $(p<$ 0.05, Figure 5D). Noteworthy, AP39 significantly downregulated hepatic HIF-1 $\alpha$ expression at mRNA and protein levels $(p<0.05$, Figure 5E). These results indicated that the adverse effects of HFD on hepatic mitochondrial dysfunction are partially reversed with AP39 treatment.

\section{Discussion}

In the present study, we demonstrate the effectiveness of AP39 in preventing HFD-induced liver injury in vivo. Treatment with AP39 alleviates obesity, hepatic steatosis, oxidative stress and mitochondrial dysfunction in HFD-fed young rats. These findings indicate that AP39 may be a promising therapeutic treatment for the prevention of pediatric NAFLD. Our finding is the first report to reveal the impact of AP39 on obesity-related liver injury.

Herein, three-week-old rats were fed with HFD for 7 weeks to mimic the symptoms of pediatric NAFLD. HFD-feeding significantly increased body weight and liver weight index, reduced food intake, elevated NAFLD activity score, ALT and AST activities and hepatic steatosis in young rats, which are known to aggravate liver 
injury and may accelerate the progression of NAFLD [27, 28]. A growing body of evidence supports that $\mathrm{H}_{2} \mathrm{~S}$ can protect against tissue damage, such as heart, liver, lung and retina [29]. AP39, a mitochondrial-targeted $\mathrm{H}_{2} \mathrm{~S}$ donor exhibits beneficial effects on tissue injury including heart, lung and neuron [17-20]. In our study, AP39 decreased body weight gain, liver weight index and liver color almost reaching normal level, as well as decreased ALT and AST activities in HFD-fed young rats. These results clearly validate that AP39 could improve HFD-induced liver injury.

Liver is a key lipid metabolic organ and abnormal lipid metabolism is tightly related to the damage of liver function. A HFD is responsible for the imbalance between lipid acquisition and disposal in the liver, which subsequently accelerates the progression of NAFLD [30]. Our results demonstrated that AP39 decreased lipid accumulation, as manifested by reduced TC, TG, LDL-C and elevated HDL-C in serum of HFD-fed rats. Previous studies have confirmed that fat accumulation in liver can block lipid metabolic homeostasis and cause severe hepatotoxicity [31]. It seems that our findings indicate the protection of AP39 on HFD-induced liver injury by maintaining the balance of lipid metabolism, although the potential mechanism by which AP39 regulates NAFLD-related lipid metabolism is still elusive until now.

A HFD results in elevated ROS production and excessive oxidative stress in liver [32], which is in line with our finding showing that ROS level was elevated in the liver of HFD-fed rats. Interestingly, in our study, AP39 suppressed HFD-induced ROS generation. Moreover, lower oxidant MDA and more antioxidants GSH and SOD are required to attenuate and repair obesity-induced liver injury [33]. Several lines of 
evidence have shown that AP39 has an antioxidant effect [16-18, 34]. Similarly, here, we found that AP39 inhibited MDA content and increased GSH level and SOD activity in HFD-fed young rats, suggesting that AP39 may ameliorate HFD-induced liver injury by inhibiting excessive oxidative stress.

Mitochondria modulate lipid metabolism and oxidative stress, and their dysfunction can lead to the disruption of lipid homeostasis, resulting in the occurrence of liver diseases including NAFLD [32, 35]. mtDNA is a major target of ROS, and HFD-feeding may damage mtDNA and aggravate liver injury [36-38]. Here, we observed a significant reduction in mtDNA quantity in the liver of HFD-fed rats, which was reversed by AP39. Besides, HFD-feeding increased the degree of mitochondrial swelling and the loss of MMP, which are similar to previous studies [25, 39]. Notably, AP39 restored hepatic mitochondrial function. Our findings were consistent with the research that AP39 protects against the loss of mtDNA and preserves mitochondrial activity $[14,18]$. The above results manifest that AP39 protects mitochondrial function in HFD-induced liver injury. More importantly, mtDNA copy number is correlated with the expression of HIF-1 $\alpha$ in liver [40]. $H I F-1 \alpha$, a key transcription factor, has been described as an important regulator of mitochondrial biogenesis, which can affect the development and progression of NAFLD [41, 42]. Covarrubias et al.'s study revealed that AP39 treatment reduces HIF-1 $\alpha$ level and improves mitochondrial function [16]. In the present study, we found that HFD-feeding upregulated HIF-1 $\alpha$ expression in the liver, while AP39 abolished it, which further confirmed the beneficial effect of AP39 on HFD-induced 
liver injury.

The pathogenesis of NAFLD is complicated and involves glucose and lipid metabolism, oxidative stress, mitochondrial damage and inflammation. HFD can increase hepatic lipid influx and de novo lipogenesis and impair insulin signaling, thus promoting triglyceride accumulation in liver and ultimately NAFLD [43]. Lensu et al.'s study found that HFD-feeding reduces food intake and enhances body weight gain and body fat content of rats [44], which is consistent with our findings. Lipid accumulation in the liver in NAFLD may be due to an imbalance in lipid metabolism as characterized by decreased oxidative capacity or increased lipogenic activity. In addition, metabolic imbalance leads to mitochondrial functional overload, which ultimately causes liver injury. Moreover, in fat-engorged hepatocytes, several vicious cycles involving ROS, lipid peroxidation products alter respiratory chain polypeptides and mtDNA, thus partially blocking the flow of electrons in the respiratory chain. Overreduction of upstream respiratory chain complexes increases mitochondrial ROS. Oxidative stress increases the release of lipid peroxidation products and inflammatory cytokines, which together trigger the liver lesions of NAFLD [45]. Therefore, in our study, HFD-feeding increased weight gain, reduced food intake of young rats, possibly due to the enhancement of oxidative stress/mitochondrial dysfunction in NAFLD. Of note, AP39 partially ameliorates the liver damage caused by HFD, indicating the protection of AP39 on HFD-induced liver injury in young rats.

In conclusion, AP39 exerts a beneficial effect on HFD-induced liver injury in young rats possibly by modulating lipid metabolism, oxidative stress and 
292

293

294

295

296

297

298

299

300

301

302

303

304

305

mitochondrial function. Current and future investigation on the effects of AP39 may provide a novel insight into the treatment of pediatric NAFLD.

\section{Author's contributions}

L.Y. designed the research study. Y.Y. and S.Y. performed the experiments. Y.Y. and D.L. analyzed the data. Y.Y. drafted the article. L.Y. revised the article. All authors read and approved the manuscript.

\section{Data availability statement}

The data used to support the findings of this study are included within the manuscript.

\section{Disclosure statement}

The authors all claim no conflicts of interest.

\section{Funding}

This work was supported by the Clinical Research Cultivation Program of The Second Affiliated Hospital of Anhui Medical university (No.2020LCYB07) and the Quality Engineering Project of Universities in Anhui Province (No. 2019jyxm1012).

\section{References}

1. Matteoni C A, Younossi Z M, Gramlich T, Boparai N, Liu Y C, McCullough A J, Nonalcoholic fatty liver disease: a spectrum of clinical and pathological severity, Gastroenterology 116(6) (1999) 1413-9. 
2. Tiniakos D G, Vos M B, Brunt E M, Nonalcoholic fatty liver disease: pathology and pathogenesis, Annual review of pathology 5 (2010) 145-71.

3. Cobbina E, Akhlaghi F, Non-alcoholic fatty liver disease (NAFLD) - pathogenesis,

4. Xu L, Nagata N, Ota T, Impact of Glucoraphanin-Mediated Activation of Nrf2 on Non-Alcoholic Fatty Liver Disease with a Focus on Mitochondrial Dysfunction, International journal of molecular sciences 20(23) (2019).

5. Kumar S, Kaufman T, Childhood obesity, Panminerva medica 60(4) (2018) 200-212.

6. Takahashi Y, Sugimoto K, Inui H, Fukusato T, Current pharmacological therapies for nonalcoholic fatty liver disease/nonalcoholic steatohepatitis, World journal of gastroenterology 21(13) (2015) 3777-85.

7. Olas B, Hydrogen sulfide in signaling pathways, Clinica chimica acta; international journal of clinical chemistry 439 (2015) 212-8.

8. Ge X, Sun J, Fei A, Gao C, Pan S, Wu Z, Hydrogen sulfide treatment alleviated ventilator-induced lung injury through regulation of autophagy and endoplasmic reticulum stress, International journal of biological sciences 15(13) (2019) $2872-2884$.

9. Wang R, Physiological implications of hydrogen sulfide: a whiff exploration that blossomed, Physiological reviews 92(2) (2012) 791-896.

10. Wen Y D, Wang H, Kho S H, Rinkiko S, Sheng X, Shen H M, et al., Hydrogen 
sulfide protects HUVECs against hydrogen peroxide induced mitochondrial dysfunction and oxidative stress, PloS one 8(2) (2013) e53147.

11. Mani S, Cao W, Wu L, Wang R, Hydrogen sulfide and the liver, Nitric oxide : biology and chemistry 41 (2014) 62-71.

12. Ren R, Yang Z, Zhao A, Huang Y, Lin S, Gong J, et al., Sulfated polysaccharide from Enteromorpha prolifera increases hydrogen sulfide production and attenuates non-alcoholic fatty liver disease in high-fat diet rats, Food \& function 9(8) (2018) 4376-4383.

13. Tan G, Pan S, Li J, Dong X, Kang K, Zhao M, et al., Hydrogen sulfide attenuates carbon tetrachloride-induced hepatotoxicity, liver cirrhosis and portal hypertension in rats, PloS one 6(10) (2011) e25943.

14. Szczesny B, Módis K, Yanagi K, Coletta C, Le Trionnaire S, Perry A, et al., AP39, a novel mitochondria-targeted hydrogen sulfide donor, stimulates cellular bioenergetics, exerts cytoprotective effects and protects against the loss of mitochondrial DNA integrity in oxidatively stressed endothelial cells in vitro, Nitric oxide : biology and chemistry 41 (2014) 120-30.

15. Ahmad A, Olah G, Szczesny B, Wood M E, Whiteman M, Szabo C, AP39, A Mitochondrially Targeted Hydrogen Sulfide Donor, Exerts Protective Effects in Renal Epithelial Cells Subjected to Oxidative Stress in Vitro and in Acute Renal Injury in Vivo, Shock (Augusta, Ga.) 45(1) (2016) 88-97.

16. Covarrubias A E, Lecarpentier E, Lo A, Salahuddin S, Gray K J, Karumanchi S A, et al., AP39, a Modulator of Mitochondrial Bioenergetics, Reduces 
Antiangiogenic Response and Oxidative Stress in Hypoxia-Exposed Trophoblasts: Relevance for Preeclampsia Pathogenesis, The American journal of pathology 189(1) (2019) 104-114.

17. Karwi Q G, Bornbaum J, Boengler K, Torregrossa R, Whiteman M, Wood M E, et

20. Ahmad A, Szabo C, Both the H(2)S biosynthesis inhibitor aminooxyacetic acid and the mitochondrially targeted $\mathrm{H}(2) \mathrm{S}$ donor AP39 exert protective effects in a mouse model of burn injury, Pharmacological research 113(Pt A) (2016) 348-355.

21. Shi T, Yang X, Zhou H, Xi J, Sun J, Ke Y, et al., Activated carbon $\mathrm{N}$-acetylcysteine microcapsule protects against nonalcoholic fatty liver disease in young rats via activating telomerase and inhibiting apoptosis, PloS one 13(1) (2018) e0189856. 
22. Yin Z, Murphy M C, Li J, Glaser K J, Mauer A S, Mounajjed T, et al., Prediction of nonalcoholic fatty liver disease (NAFLD) activity score (NAS) with multiparametric hepatic magnetic resonance imaging and elastography, European radiology 29(11) (2019) 5823-5831.

23. Wang W, Wang M, Ruan Y, Tan J, Wang H, Yang T, et al., Ginkgolic Acids Impair Mitochondrial Function by Decreasing Mitochondrial Biogenesis and Promoting FUNDC1-Dependent Mitophagy, Journal of agricultural and food chemistry 67(36) (2019) 10097-10106.

24. Jia N, Lin X, Ma S, Ge S, Mu S, Yang C, et al., Amelioration of hepatic steatosis is associated with modulation of gut microbiota and suppression of hepatic miR-34a in Gynostemma pentaphylla (Thunb.) Makino treated mice, Nutrition \& metabolism 15 (2018) 86.

25. Ren T, Zhu L, Shen Y, Mou Q, Lin T, Feng H, Protection of hepatocyte mitochondrial function by blueberry juice and probiotics via SIRT1 regulation in non-alcoholic fatty liver disease, Food \& function 10(3) (2019) 1540-1551.

26. Mann J P, Valenti L, Scorletti E, Byrne C D, Nobili V, Nonalcoholic Fatty Liver Disease in Children, Seminars in liver disease 38(1) (2018) 1-13.

27. Xia H M, Wang J, Xie X J, Xu L J, Tang S Q, Green tea polyphenols attenuate hepatic steatosis, and reduce insulin resistance and inflammation in high-fat diet-induced rats, International journal of molecular medicine 44(4) (2019) 1523-1530.

28. Kitade H, Chen G, Ni Y, Ota T, Nonalcoholic Fatty Liver Disease and Insulin 
29. Wu D, Wang J, Li H, Xue M, Ji A, Li Y, Role of Hydrogen Sulfide in Ischemia-Reperfusion Injury, Oxidative medicine and cellular longevity 2015

30. Ipsen D H, Lykkesfeldt J, Tveden-Nyborg P, Molecular mechanisms of hepatic

32. Sumida Y, Niki E, Naito Y, Yoshikawa T, Involvement of free radicals and oxidative stress in NAFLD/NASH, Free radical research 47(11) (2013) 869-80.

33. Zhang L, Li H X, Pan W S, Ullah Khan F, Qian C, Qi-Li F R, et al., Administration of methyl palmitate prevents non-alcoholic steatohepatitis (NASH) by induction of PPAR- $\alpha$, Biomedicine $\&$ pharmacotherapy $=$ Biomedecine $\&$ pharmacotherapie 111 (2019) 99-108.

34. Gerő D, Torregrossa R, Perry A, Waters A, Le-Trionnaire S, Whatmore J L, et al., The novel mitochondria-targeted hydrogen sulfide $(\mathrm{H}(2) \mathrm{S})$ donors AP123 and AP39 protect against hyperglycemic injury in microvascular endothelial cells in vitro, Pharmacological research 113(Pt A) (2016) 186-198.

35. Paradies G, Paradies V, Ruggiero F M, Petrosillo G, Oxidative stress, cardiolipin 
and mitochondrial dysfunction in nonalcoholic fatty liver disease, World journal of gastroenterology 20(39) (2014) 14205-18.

36. Jha S K, Jha N K, Kumar D, Ambasta R K, Kumar P, Linking mitochondrial dysfunction, metabolic syndrome and stress signaling in Neurodegeneration, Biochimica et biophysica acta. Molecular basis of disease 1863(5) (2017) 1132-1146.

37. Malik A N, Czajka A, Is mitochondrial DNA content a potential biomarker of mitochondrial dysfunction?, Mitochondrion 13(5) (2013) 481-92.

38. Malik A N, Simões I C M, Rosa H S, Khan S, Karkucinska-Wieckowska A, Wieckowski M R, A Diet Induced Maladaptive Increase in Hepatic Mitochondrial DNA Precedes OXPHOS Defects and May Contribute to Non-Alcoholic Fatty Liver Disease, Cells 8(10) (2019).

39. Simões I C M, Fontes A, Pinton P, Zischka H, Wieckowski M R, Mitochondria in non-alcoholic fatty liver disease, The international journal of biochemistry \& cell biology 95 (2018) 93-99.

40. Chowdhury A, Aich A, Jain G, Wozny K, Lüchtenborg C, Hartmann M, et al., Defective Mitochondrial Cardiolipin Remodeling Dampens HIF-1 $\alpha$ Expression in Hypoxia, Cell reports 25(3) (2018) 561-570.e6.

41. Gonzalez F J, Xie C, Jiang C, The role of hypoxia-inducible factors in metabolic diseases, Nature reviews. Endocrinology 15(1) (2018) 21-32.

42. Carabelli J, Burgueño A L, Rosselli M S, Gianotti T F, Lago N R, Pirola C J, et al., High fat diet-induced liver steatosis promotes an increase in liver mitochondrial 

15(6) (2011) 1329-38.

43. Antonucci L, Porcu C, Iannucci G, Balsano C, Barbaro B, Non-Alcoholic Fatty Liver Disease and Nutritional Implications: Special Focus on Copper, Nutrients gastroenterology and hepatology 22 Suppl 1 (2007) S20-7.

Table 1. Sequence of primers used in RT-qPCR.

\begin{tabular}{lll}
\hline Gene & Primers & Sequence (5'-3') \\
\hline mtDNA & Forward & TGAGCCATCCCTTCACTAGG \\
& Reverse & TGAGCCGCAAATTTCAGAG \\
nuclear DNA ( $\beta$-actin) & Forward & CTGCTCTTTCCCAGATGAGG \\
& Reverse & CCACAGCACTGTAGGGGTTT \\
HIF-1 & Forward & CTACTATGTCGCTTTCTTGG \\
& Reverse & GTTTCTGCTGCCTTGTATGG \\
$\boldsymbol{\beta}$-actin & Forward & GGAGATTACTGCCCTGGCTCCTAGC \\
& Reverse & GGCCGGACTCATCGTACTCCTGCTT \\
\hline
\end{tabular}


$\mathbf{A}$

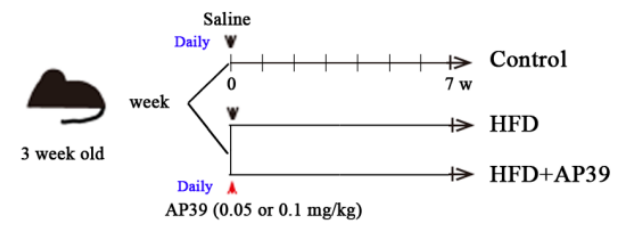

456

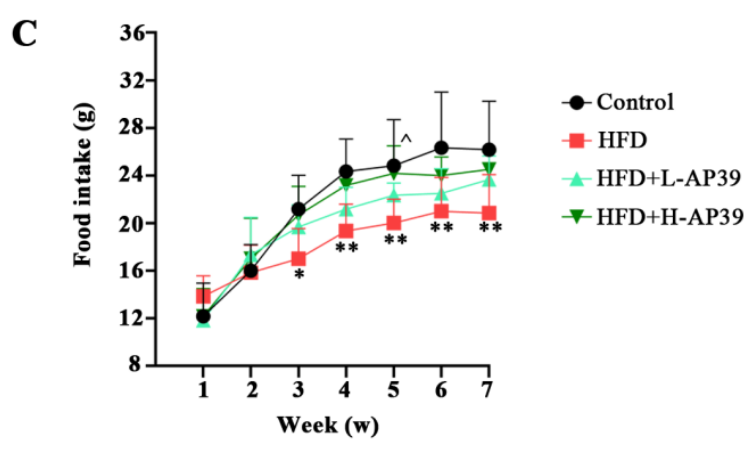

B

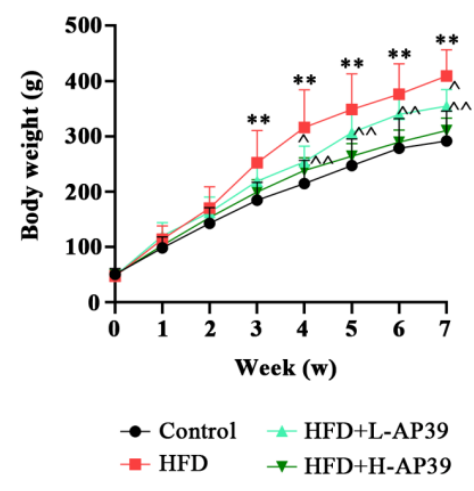

457 Figure 1. Effect of AP39 on obesity. High-fat-diet (HFD)-induced NAFLD rats were

458 intravenously injected with 0.05 or $0.1 \mathrm{mg} / \mathrm{kg}$ AP39 once a day for 7 weeks. (A)

459 Schematic abstract of the experimental process. (B-C) Body weight and food intake of 460 young rats was recorded once a week for 7 weeks. ${ }^{* *} p<0.01$ versus Control; ${ }^{\wedge} p<0.05$, 461 ${ }^{\wedge} p<0.01$ versus HFD. 
$\mathbf{A}$

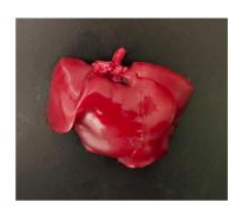

Control

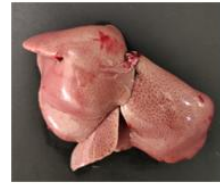

HFD+L-AP39

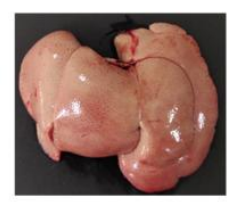

HFD

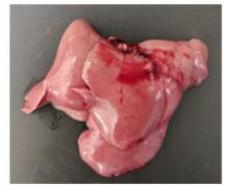

HFD+H-AP39

C
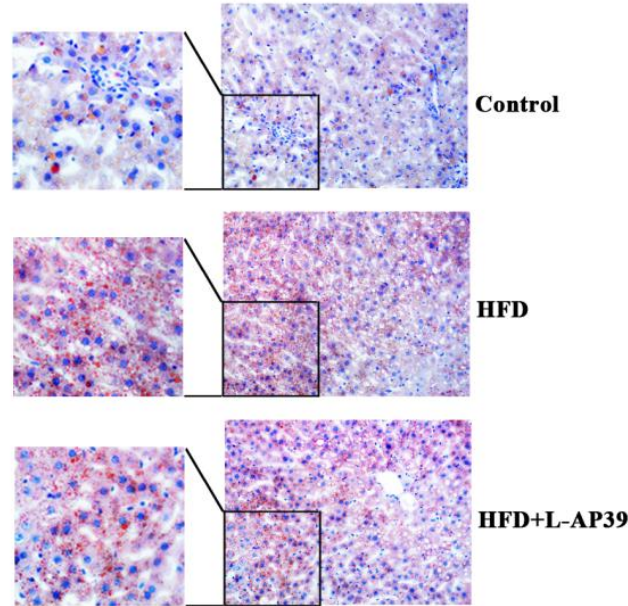

B

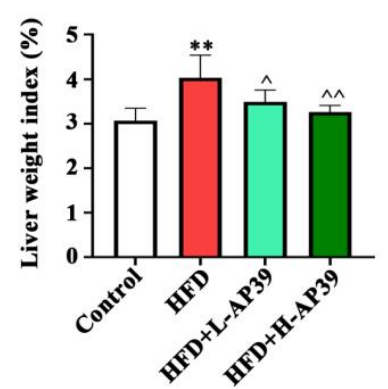

D

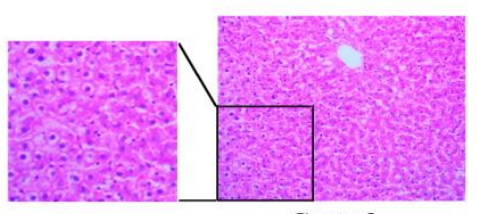

Control

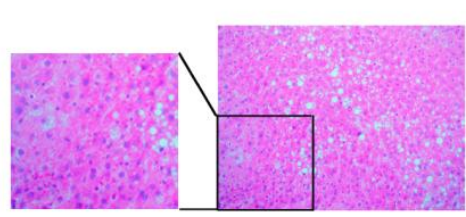

HFD+L-AP39

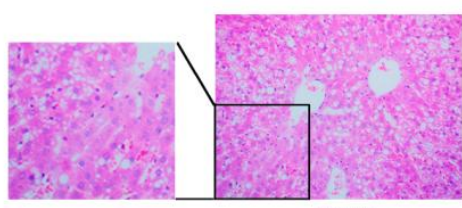

HFD

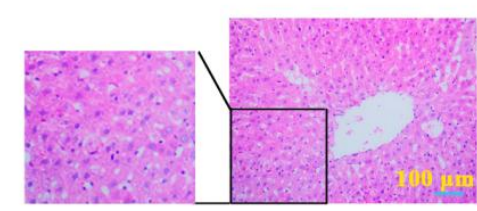

HFD+H-AP39
$\mathbf{E}$

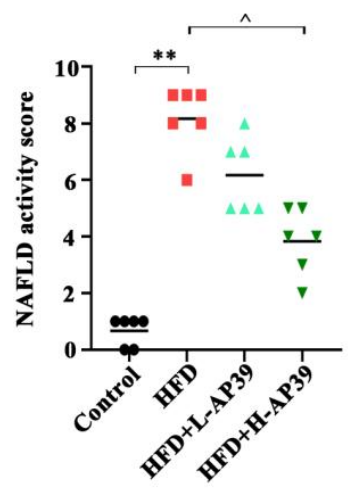

Figure 2. Effect of AP39 on HFD-induced NAFLD. (A) At the end of the experiment, the liver tissues were harvested. Gross morphology of liver tissue was observed. (B) Liver weight index was calculated according to the formula liver wet weight / body weight $\times 100 \%$. (C-D) The histological alternations of liver tissues were visualized with Oil red O staining $(\times 200)$ and H\&E staining $(\times 200)$, and the representative photomicrographs were shown. (E) NAFLD activity score, a histological scoring system for NAFLD, was counted. ${ }^{* *} p<0.01$ versus Control; ${ }^{\wedge} p<0.05,{ }^{\wedge} p<0.01$ 
$\mathbf{A}$

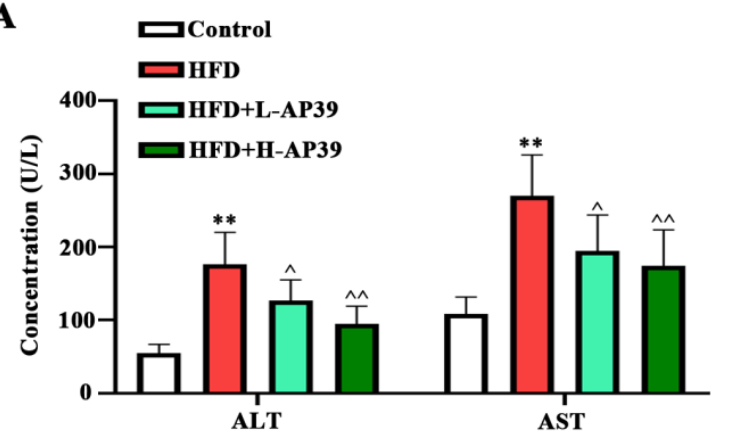

$\mathbf{C}$

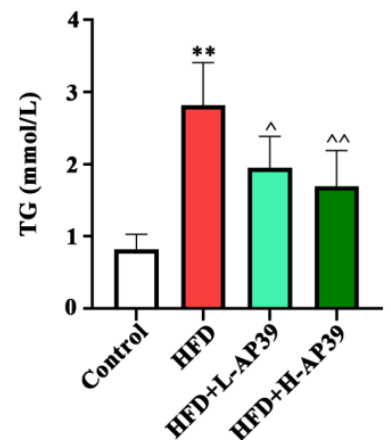

D

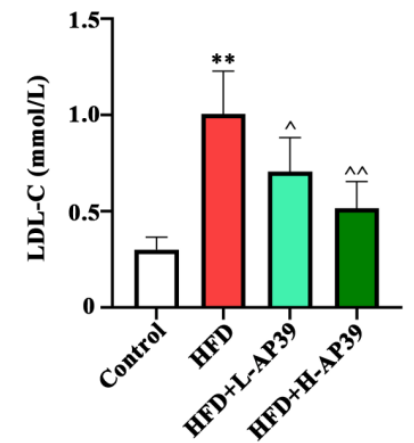

B

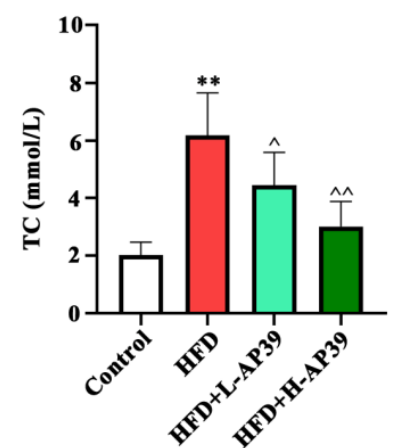

$\mathbf{E}$

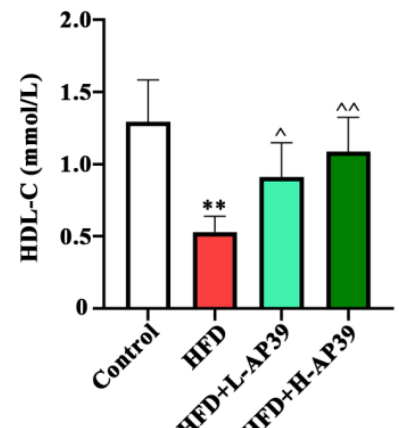

472 Figure 3. Effect of AP39 on lipid profiles in serum of rats. (A-E) Relative levels of

473 serum ALT, AST, TC, TG, LDL-C and HDL-C were determined using the 474 corresponding kits. ALT, alanine transaminase; AST, aspartate transaminase; TC, total 475 cholesterol; TG, total triglycerides; LDL-C, low-density lipoprotein cholesterol; 476 HDL-C, high-density lipoprotein cholesterol. ${ }^{* *} p<0.01$ versus Control; $\stackrel{\wedge}{p}<0.05,{ }^{\wedge} p$ $<0.01$ versus HFD. 
$\mathbf{A}$

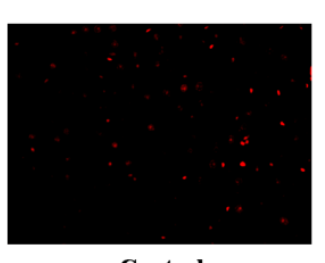

Control

B

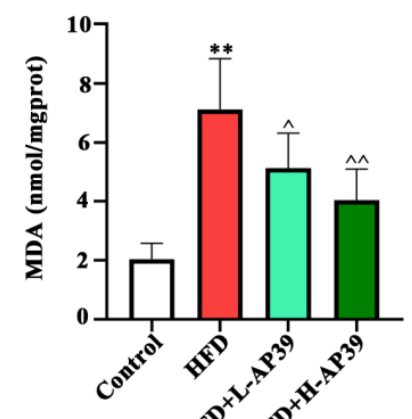

C

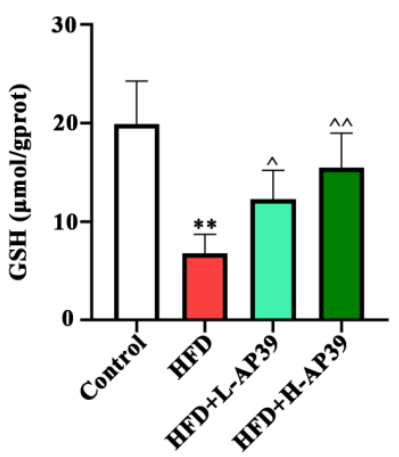

D

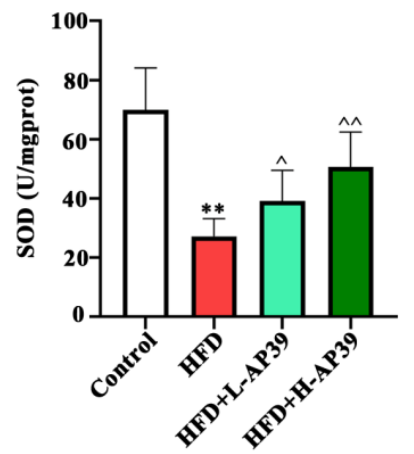

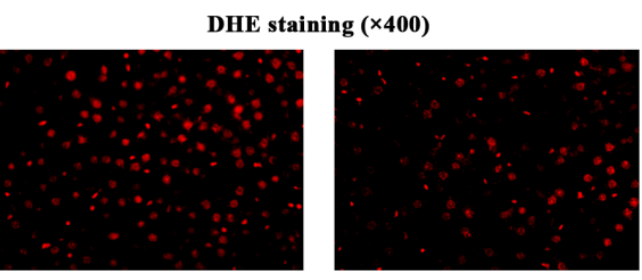

HFD+L-AP39

HFD

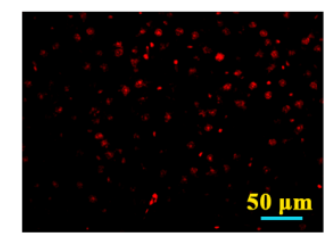

HFD+H-AP39

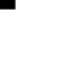


A

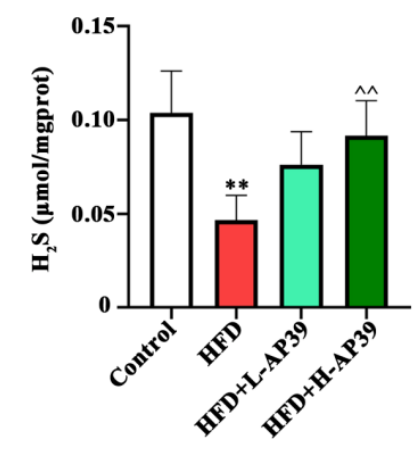

D

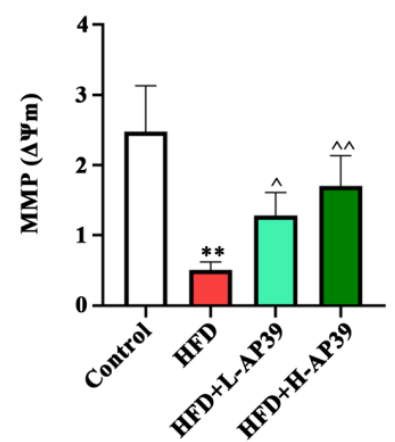

B

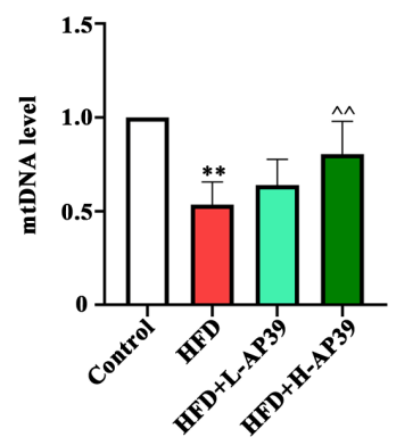

$\mathbf{E}$

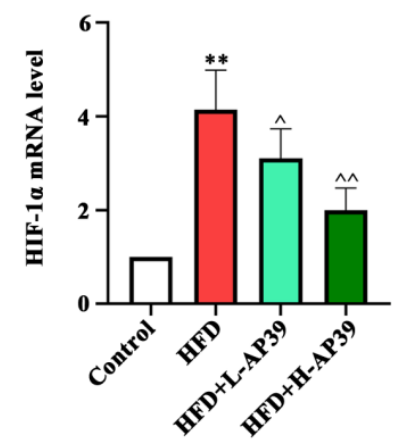

C
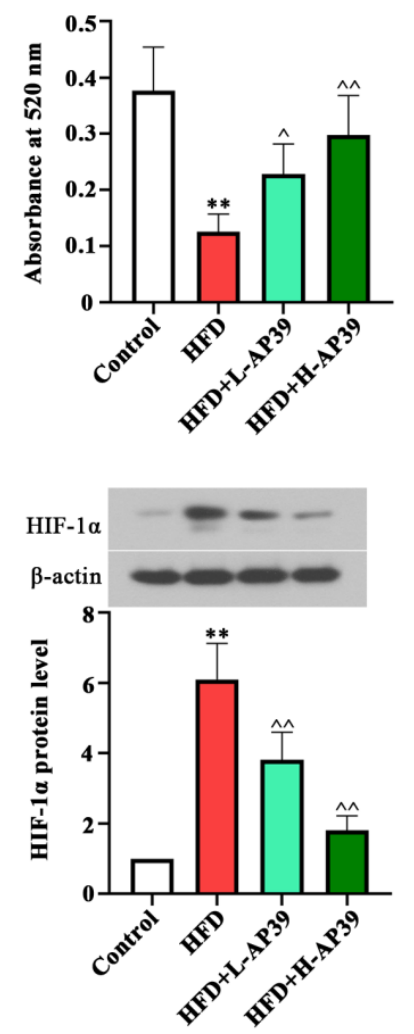

Figure 5. Effect of AP39 on $\mathrm{H}_{2} \mathrm{~S}$ level and mitochondrial function. (A) Hepatic $\mathrm{H}_{2} \mathrm{~S}$ level was detected using the $\mathrm{H}_{2} \mathrm{~S}$ determination kit. (B) mtDNA copy number in the liver was measured by RT-qPCR. (C) The degree of mitochondrial swelling was analyzed at $520 \mathrm{~nm}$ wavelength on a spectrophotometer. (D) Mitochondrial membrane potential (MMP) changes were detected using JC-1 probe. (E) Hypoxia-inducible factor-1 $\alpha(\mathrm{HIF}-1 \alpha)$ expression at mRNA and protein levels was determined by

491 RT-qPCR and western blot, respectively. ${ }^{* *} p<0.01$ versus Control; ${ }^{\wedge} p<0.05,{ }^{\wedge} p<$ 0.01 versus HFD. 